\title{
High-power Ultrafast Solid-state Laser Using Graphene Based Saturable Absorber
}

\author{
Z. Sun ${ }^{1 *}$, X. C. Lin $^{2}$, H. J. Yu ${ }^{2}$, T. Hasan ${ }^{1}$, F. Torrisi ${ }^{1}$, L. Zhang ${ }^{2}$, L. Sun ${ }^{2}$, \\ L. Guo ${ }^{2}$, W. $\mathrm{Hou}^{2}$, J.M. Li' $\mathbf{i}^{2}$, and A. C. Ferrari ${ }^{1}$ \\ 1Department of Engineering, University of Cambridge, Cambridge, CB3 OFA, United Kingdom \\ 2Laboratory of All-solid-state Light Sources, Institute of Semiconductors, Beijing, 100083, China \\ E-mail: zs244@cam.ac.uk
}

\begin{abstract}
We demonstrate a graphene based saturable absorber mode-locked Nd: $\mathrm{YVO}_{4}$ solid-state laser, generating $\sim 14 \mathrm{~nJ}$ pulses with $\sim 1 \mathrm{~W}$ average output power. This shows the potential for high-power pulse generation.

(C) 2011 Optical Society of America

OCIS codes: (320.7090) Ultrafast lasers; (140.3580) Lasers, solid-state; (160.4330) Nonlinear optical materials.
\end{abstract}

\section{Introduction}

Passively mode-locked solid-state lasers are the main work-horse for various applications in industry, research and military [1-2]. Currently, high-power ultrafast solid-state lasers are mainly mode-locked by semiconductor saturable absorber mirrors (SESAMs)[1-3]. These devices require expensive and complex epitaxial growth techniques (e.g. molecular beam epitaxy)[2-3] and subsequent post-processing to reduce recovery time[2-3]. Single wall carbon nanotubes[4-13] and more recently, graphene [13-19] based saturable absorbers have attracted great attention for ultrafast pulse generation, due to their unique properties, such as sub-picosecond recovery time, broadband operation, low saturation intensity, easy fabrication and integration. In particular, graphene based saturable absorbers (GSA) are attractive SAs because they do not require bandgap engineering [13-25], due to the linear band dispersions [20]. After the first demonstration of graphene mode-locking [13], most efforts on GSA focussed on fiber laser mode-locking [13-19], while very few groups reported graphene mode-locked solid-state lasers[21-22]. Thus far, for graphene mode-locked solid-state lasers, the maximum output pulse energy and average power are $\sim 1 \mathrm{~nJ}$ and $100 \mathrm{~mW}$ [21], not exploiting the high-power capabilities of solid-state lasers.

Here, we report a high-power passively mode-locked $\mathrm{Nd}: \mathrm{YVO}_{4}$ laser using GSA, with $\sim 1 \mathrm{~W}$ average power. The repetition rate and pulse energy are $\sim 75 \mathrm{MHz}$ and $\sim 14 \mathrm{~nJ}$, respectively.

\section{Experimental Setup and Results}

Graphene dispersions are prepared using method in Ref.[17], then spin-coated on quartz to form a GSA. The absorption spectra of the GSA and a reference quartz substrate are presented in Fig.1(a). The GSA absorption is featureless[17], while the quartz substrate contributes small peaks in the infrared spectral range [26].

The GSA is then inserted in a solid-state laser, shown in Fig.1 (b). We use a $3 \times 3 \times 15 \mathrm{~mm}^{3} \mathrm{Nd}: \mathrm{YVO}_{4}$ crystal with a $\mathrm{Nd}^{3+}$-doping concentration of 0.3 at.\% as the gain medium. Both facets of the crystal are coated with an anti-reflection (AR) coating for $1064 \mathrm{~nm}$. The crystal is pumped by an 880nm fiber-coupled laser diode (LD). The fiber has a $200 \mu \mathrm{m}$ core diameter and a 0.22 numerical aperture (NA). The beam diameter of pump beam inside the crystal is $\sim 400 \mathrm{~m}$, coupled with a beam shaping telescope. M1 is a flat mirror with AR coating at 880 $\mathrm{nm}$ and high reflection (HR) coating at $1064 \mathrm{~nm}$. M2 is a wedge-shaped mirror used as an output coupler with $10 \%$ transmittance at $1064 \mathrm{~nm}$. Both have HR coating at $1064 \mathrm{~nm}$. M5 is a flat mirror with HR coating at 1064 $\mathrm{nm}$. The GSA is put between M3 and M5 for passive mode-locking. The total cavity length is $\sim 2.0 \mathrm{~m}$.

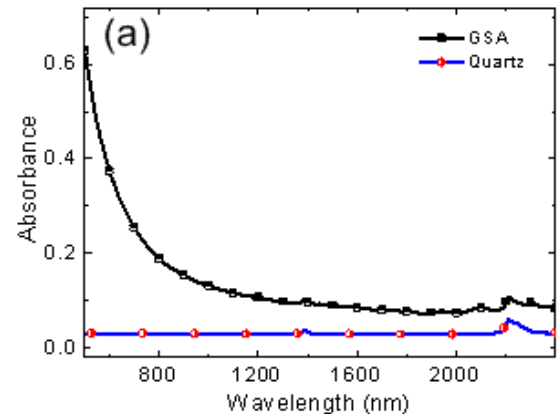

Fig. 1. (a) Absorption spectra of GSA and quartz substrate.

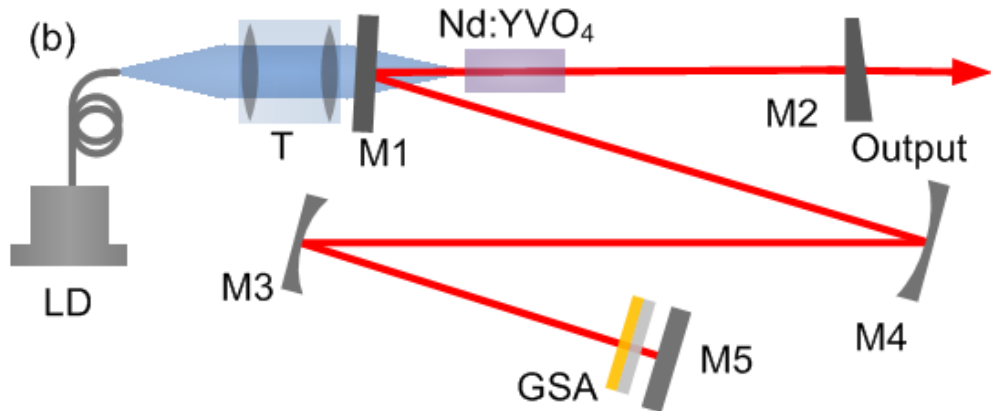

(b) Laser setup. LD: Laser diode; T: Telescope.

Continuous wave (CW) lasing threshold is $\sim 2 \mathrm{~W}$. Mode-locked pulses are observed when the pump power is increased to 3.6W. The pulses are shown in Fig.2. The pulse profile is measured using a digital oscilloscope (Agilent DSO6104A) with 1GHz bandwidth and a photodiode with 100ps rising time. As shown Fig. 2, the 
pulse interval equals to $\sim 13.3$ ns cavity round trip time, indicating mode-locking [2]. The repetition rate is $\sim 75 \mathrm{MHz}$, in agreement with the cavity design parameters. The peak output wavelength is $\sim 1064 \mathrm{~nm}$. The full width at half maximum of the output spectrum is $\sim 0.17 \mathrm{~nm}$.

The average output power increases almost linearly with the pump power. The maximum average output power we achieved is $\sim 1 \mathrm{~W}$ at $2.6 \mathrm{~W}$ pump power. The optical-to-optical conversion efficiency is $\sim 16 \%$ with a $\sim 20 \%$ slope efficiency. The corresponding pulse energy is $\sim 14 \mathrm{~nJ}$. Our output power is around one-order of magnitude higher than previous graphene mode-locked lasers [13-19,21-22].

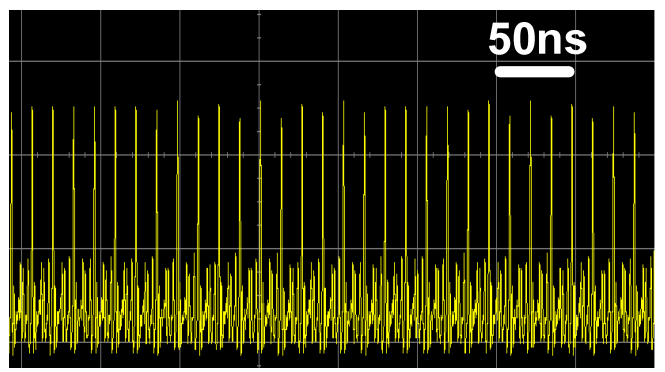

Fig. 2. Mode-locked pulse.

\section{Conclusions}

We reported a high-power passively mode-locked solid-state laser using GSA, with a $\sim 1 \mathrm{~W}$ output power and an optical-to-optical conversion efficiency of 16\%. The repetition rate and pulse energy are $\sim 75 \mathrm{MHz}$ and $\sim 14 \mathrm{~nJ}$, respectively. This work paves a way to GSA based high-power and high-efficiency ultrafast lasers.

We acknowledge funding from EPSRC grants GR/S97613/01, EP/E500935/1, ERC grant NANOPOTS, King's College, Royal Society Brian Mercer Award, Royal Society Wolfson Research Merit Award and the Cambridge Integrated Knowledge Centre.

\section{References}

[1] W. Koechner, Solid-State Laser Engineering, (Springer, New York, 2006).

[2] U. Keller, Ultrafast solid-state lasers. (Elsevier, Amsterdam, 2004).

[3] U. Keller, "Recent developments in compact ultrafast lasers," Nature 424, 831 (2003).

[4] S. Y. Set et al., in Optical Fiber Communication Conference (OFC), Vol. 87 of OSA Trends in Optics and Photonics (Optical Society of America, 2003), postdeadline paper PD44.

[5] F. Wang et al.,"Wideband-tuneable, nanotube mode-locked, fibre laser," Nat. Nanotechnol. 3, 738 (2008).

[6] S. Kivistö et al., "Carbon nanotube films for ultrafast broadband technology," Opt. Express 17, 2358-2363 (2009).

[7] Z. Sun et.al., "A compact, high power, ultrafast laser mode-locked by carbon nanotubes," Appl. Phys. Lett. 95, 253102 (2009)

[8] K. H. Fong et.al.,"Solid-state Er : Yb : glass laser mode-locked by using single-wall carbon nanotube thin film," Opt. Lett. 32, 38(2007).

[9] V. Scardaci et al., "Carbon Nanotube Polycarbonate Composites for Ultrafast Lasers." Adv. Mater. 20, 4040 (2008).

[10] W. Cho et.al.,"Boosting the Nonlinear Optical Response of Carbon Nanotube Saturable Absorbers,"Adv. Funct. Mater.20,1937 (2010).

[11] E. J. R. Kelleher et al., "Nanosecond-pulse fiber lasers mode-locked with nanotubes," Appl. Phys. Lett. 95, 111108 (2009).

[12] Z. Sun et.al., "Ultrafast Stretched-Pulse Fiber Laser Mode-Locked by Carbon Nanotubes," Nano Res. 3, 404 (2010).

[13]T. Hasan et.al., "Nanotube-Polymer Composites for Ultrafast Photonics," Adv. Mater. 21, 3874 (2009).

[14] Z. Sun et.al., "Graphene Mode-Locked Ultrafast Laser," ACS Nano 4, 803(2010).

[15] Z. Sun et.al., "A stable, wideband tunable, near transform-limited, graphene-mode-locked, ultrafast laser," Nano Res. 3, 653(2010),

[16] Y.Song et.al.,"Graphene mode-lockers for fiber lasers functioned with evanescent field interaction" Appl. Phys. Lett. 96,051122(2010).

[17] F. Bonaccorso et.al., "Graphene Photonics and Optoelectronics," Nat. Photonics 4, 611(2010).

[18] A. Martinez et.al.,"Optical deposition of graphene and carbon nanotubes in a fiber ferrule," Opt. Express18,23054 (2010).

[19] D. Popa et.al., "Sub 200fs pulse generation from a graphene mode-locked fiber laser," Appl. Phys. Lett. 97, 203106 (2010).

[20] A. K. Geim et al., "The rise of graphene," Nat. Mater. 6,183(2007).

[21] W. Tan et.al., "Mode locking of ceramic Nd:yttrium aluminum garnet with graphene " Appl. Phys. Lett. 96, 031106 (2010).

[22] C. Lee et.al., "Ultra-Short Optical Pulse Generation with Single-Layer Graphene," J. Nonlinear Opt. Phys. Mater., In press (2010).

[23] H. Yu et.al. "Large Energy Pulse Generation Modulated by Graphene Epitaxially Grown on Silicon Carbide", ACS Nano, DOI: $10.1021 / \mathrm{nn} 102280 \mathrm{~m}(2010)$

[24] Y. M. Chang et.al., "Multilayered graphene efficiently formed by mechanical exfoliation for nonlinear saturable absorbers in fiber mode-locked lasers" Appl. Phys. Lett. 97,211102 (2010).

[25] D. Popa et.al., "Graphene Q-switched, tunable fiber laser", arXiv, 1011.0115v1 (2010).

[26] J. H. Simmons and K. S. Potter, Optical Materials (Academic Press, 2000). 